\section{Reconciling Neuroimaging and Clinical Findings in Aicardi-Goutières Syndrome: An Autoimmune-Mediated Encephalopathy}

In the November-December 2009 issue of the American Journal of Neuroradiology, Uggetti et $\mathrm{al}^{1}$ presented neuroradiologic findings in 36 patients with Aicardi-Goutières syndrome (AGS), a genetically determined early-onset encephalopathy resembling congenital viral infection characterized by basal ganglia calcifications, white matter loss, and brain atrophy. This study represents the first comprehensive case series with a detailed description of neuroimaging findings in AGS. While the primary focus of this study was the brain, the authors did not mention important clinical and genetic aspects that are critical to our current understanding of AGS as an autoimmune-mediated disorder.

We recently reported a series of 20 patients with molecularly confirmed AGS and showed that features of the autoimmune disease systemic lupus erythematosus (SLE) are much more prevalent than previously assumed. ${ }^{2}$ Thus, in addition to erythematous lesions at acral locations also referred to as "chilblain lesions," these features included arthritis, oral ulcers, thrombocytopenia, leukocytopenia, antinuclear antibodies, and complement deficiency. ${ }^{2}$ Consistent with the report by Uggetti et al, neuroradiologic features in our cohort encompassed intracranial calcifications, loss of white matter, and atrophic changes that were observed in all patients, albeit with great variability. Similarly, the extent of neuroimaging pathology correlated with the age of onset and the degree of neurologic disease, such as tetraparesis, dystonia, and developmental delay often in the context of epilepsy.

Cerebral calcifications primarily involving the basal ganglia and the periventricular white matter constitute a landmark of AGS and present either as a few calcified spots or confluent symmetric lesions extending into the deep white matter. ${ }^{2}$ Although cerebellar calcifications were not reported in the series of Uggetti et al, ${ }^{1}$ we also noted calcifications of the dentate nuclei in some cases as originally described by Aicardi and Goutières. ${ }^{3}$ Notably, cerebral calcifications were not always evident as susceptibility artifacts on $\mathrm{T} 2^{*}$-weighted images despite the presence of extensive calcification on CT (Fig 1). White matter abnormalities ranged from discrete hyperintensities on T2-weighted images to severe frontotemporal leukodystrophy with bitemporal vacuolar lesions in the white matter, ${ }^{2}$ a finding that was also observed in the study by Uggetti et al. Although severe frontotemporal leukodystrophy with cystlike changes was noted only in some cases, this pattern represents a highly characteristic MR imaging finding in AGS and should prompt genetic investigation.

AGS is caused by biallelic mutations in at least 5 genes encoding the deoxyribonucleic acid (DNA) exonuclease TREX1 (AGS1), the 3 subunits of the ribonuclease $\mathrm{H} 2$ (RNASEH2A/AGS4; RNASEH2B/ AGS2; RNASEH2C/AGS3), and the putative DNA binding protein SAMHD1, respectively. ${ }^{4}$ In addition, heterozygous TREX1 mutations have been reported in patients with autosomal dominant retinal vasculopathy with cerebral leukodystrophy (RVCL), sporadic SLE, and in a patient with neuropsychiatric SLE presenting with white matter lesions and cerebral vasculopathy. ${ }^{5-7}$ Most interesting, activation of the interferon- $\alpha$ axis is a finding common to AGS and SLE, indicating that impairment of intracellular nucleic acid metabolism can result in systemic autoimmunity via activation of the innate immune system. ${ }^{4}$

In view of the fact that both RVCL and SLE are characterized by vasculitic changes, it is likely that the neuroimaging findings in AGS are at least in part due to microangiopathy. Uggetti et al ${ }^{1}$ argue against a microangiopathic origin of the neuropathology in AGS on the basis

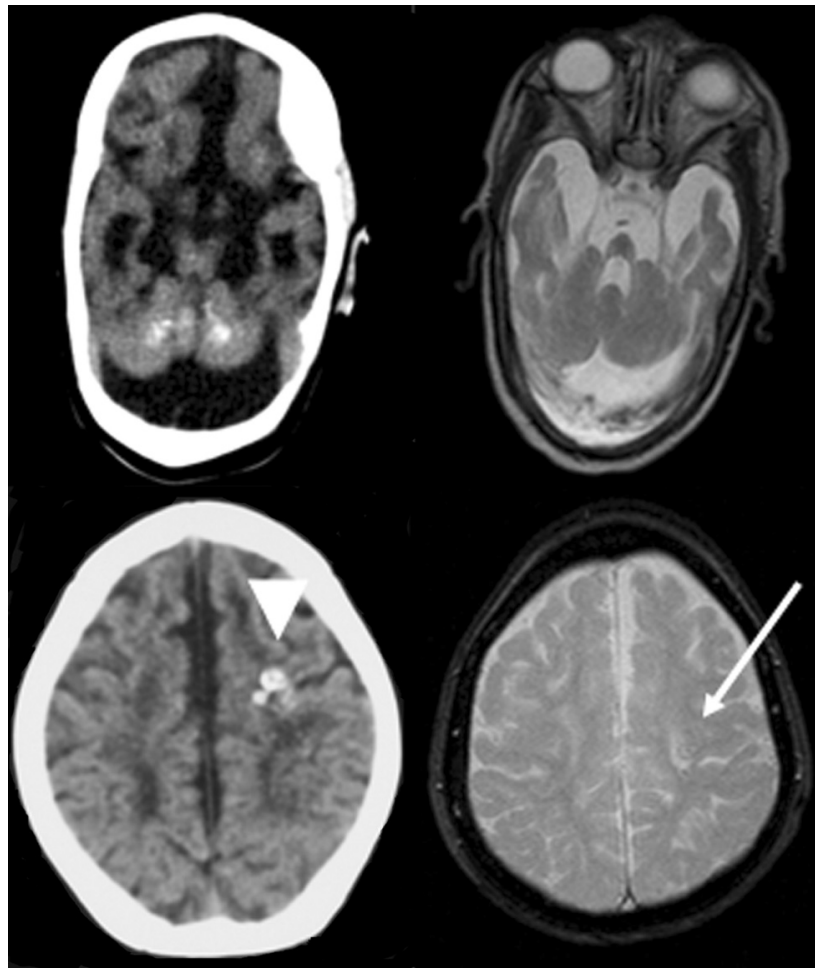

Fig 1. Upper row: CT scan and T2 turbo spin-echo image of the cerebellum. On the CT scan, diffuse calcifications in both cerebellar hemispheres are depicted. These calcifications are not visible on the T2 turbo spin-echo image. Lower row: CT scan and T2* weighted image of the cerebrum. Both images are at the level of the centrum semiovale. On the CT scan, punctuate calcinations are visible in the left frontal lobe (arrowhead). On the T2* image leukoencephalopathic changes of the white matter are depicted. A small vacuole is visible on the left side at the site of the calcification (arrow), along with susceptibility artifacts.

of the absence of contrast enhancement in any of the patients studied. This is in contrast to the findings of Barth et al, ${ }^{8}$ who reported smallvessel calcifications as well as microinfarctions consistent with microangiopathy on postmortem examination in a patient with AGS, a finding that further highlights common pathophysiologic mechanisms in AGS and SLE. ${ }^{2}$

Because early immune-modulating therapeutic intervention might be potentially beneficial in children with AGS, neuroradiologic pattern recognition is crucial during diagnostic work-up. Thus, in the absence of viral infection or metabolic disease, any child presenting with symmetric calcifications and leukodystrophy with hyperintense microangiopathic white matter lesions should be referred for genetic testing for AGS.

\section{References}

1. Uggetti C, La Piana R, Orcesi S, et al. Aicardi-Goutières syndrome: neuroradiologic findings and follow-up. AJNR Am J Neuroradiol 2009;30:1971-76. Epub 2009 Jul 23

2. Ramantani G, Kohlhase J, Hertzberg C, et al. Expanding the phenotypic spectrum of lupus erythematosus in Aicardi-Goutières syndrome. Arthritis Rheum 2010;62:1208-12

3. Aicardi J, Goutières F. A progressive familial encephalopathy in infancy with calcifications of the basal ganglia and chronic cerebrospinal fluid lymphocytosis. Ann Neurol 1984;15:49-54

4. Lee-Kirsch MA. Nucleic acid metabolism and systemic autoimmunity revisited. Arthritis Rheum 2010;62:1469-77

5. Richards A, van den Maagdenberg AM, Jen JC, et al. C-terminal truncations in human $3^{\prime}-5$ ' DNA exonuclease TREX1 cause autosomal dominant retinal vasculopathy with cerebral leukodystrophy. Nat Genet 2007;39:1068-70 Epub 2007 Jul 29

6. Lee-Kirsch MA, Gong M, Chowdhury D, et al. Mutations in the gene encoding the $3^{\prime}-5^{\prime}$ DNA exonuclease TREX1 are associated with systemic lupus erythematosus. Nat Genet 2007;39:1065-67 
7. de Vries B, Steup-Beekman G, Haan J, et al. TREX1 gene variant in neuropsychiatric systemic lupus erythematosus. Ann Rheum Dis 2010 Apr 13. [Epub ahead of print]

8. Barth PG, Walter A, van Gelderen I. Aicardi-Goutières syndrome: a genetic microangiopathy? Acta Neuropathol 1999;98:212-16

G. Ramantani Epilepsy Center

University Hospital of Freiburg

Freiburg, Germany

P. Niggemann

Privatpraxis für Kernspintomographie

Köln-Rodenkirchen, Germany

T. Bast

Epilepsiezentrum Kork

Kehl-Kork, Germany

M.A. Lee-Kirsch

Klinik und Poliklinik für Kinder- und Jugendmedizin

Technische Universität

Dresden, Germany

DOI 10.3174/ajnr.A2147 